\title{
Statistical indicators and state-space population models predict extinction in a population of bobwhite quail
}

\author{
Trevor J. Hefley • Andrew J. Tyre • Erin E. Blankenship
}

Received: 13 February 2013 / Accepted: 4 June 2013 / Published online: 12 July 2013

(C) The Author(s) 2013. This article is published with open access at Springerlink.com

\begin{abstract}
Early warning systems of extinction thresholds have been developed for and tested in microcosm experiments, but have not been applied to populations of wild animals. We used state-space population models and a statistical indicator to detect a transcritical bifurcation extinction threshold in a population of bobwhite quail (Colinus virginianus) located in an agricultural region experiencing habitat deterioration and loss. The extinction threshold was detectible using two independent data sets. We compared predictions from state-space population models to predictions from a statistical indicator and found that predictions were corroborated. Using state-space population models, we estimated that our study population crossed the extinction threshold in 2010 (2002-2036; $95 \%$ confidence intervals [CI]) using the whistle count (WC) data set and in 2008 (1999-2064; 95 \% CI) using the Breeding Bird Survey (BBS) data. With the statistical indicator, we estimated that the extinction threshold will be crossed in 2018 (2004-2031; $95 \%$ CI) using the WC data and will be crossed in 2012 (2006-2018; $95 \% \mathrm{CI}$ ) using the BBS data. We expect extinction in our study population soon after crossing the extinction threshold, but the time to extinction and potential reversibility of the threshold are unknown. Our results suggest that neither small nor decreasing population size will warn of the transcritical bifurcation extinction threshold. We suggest that managers of wildlife populations in regions experiencing land use change should try to
\end{abstract}

T. J. Hefley $(\bowtie) \cdot$ E. E. Blankenship

Department of Statistics, University of Nebraska-Lincoln, 3310

Holdrege Street, Lincoln, NE 68583, USA

e-mail: thefley@huskers.unl.edu

E. E. Blankenship

e-mail: erin.blankenship@unl.edu

T. J. Hefley $\cdot$ A. J. Tyre

School of Natural Resources, University of Nebraska-Lincoln,

3310 Holdrege Street, Lincoln, NE 68583, USA

A. J. Tyre

e-mail: atyre2@unl.edu predict extinction thresholds and make management decisions to ensure the persistence of the species.

Keywords Bobwhite quail - Colinus virginianus . Extinction - State-space model $\cdot$ Statistical indicator . Transcritical bifurcation

\section{Introduction}

Understanding and predicting extinction are central concerns of conservation biology. Much focus has been given to the effects of demographic and environmental stochasticity on population extinction (Melbourne and Hastings 2008), forecasting quasi-extinction probabilities (Holmes et al. 2007), and population viability analyses (Nadeem and Lele 2012) with less focus directed on identifying and forecasting critical threshold crossings that result in transitions to alternative population states including extinction (Wissel 1984; Abrams 2002; Drake and Griffen 2010; Dai et al. 2012, 2013). This shortage of research and application is particularly evident in the management of declining populations, where it is often assumed that a decreasing or small population size warns of future extinction (International Union for Conservation of Nature 2001; Abrams 2002). If threshold dynamics occur, a population with a slowly declining, stable, or even an increasing trend in abundance could collapse rapidly to extinction even when the trends in the environmental variables driving the population over the extinction threshold remain constant (Wissel 1984; Abrams 2002; Drake and Griffen 2010; Dai et al. 2012, 2013).

Dynamical systems theory shows that climate, physiological, and ecological thresholds can be detected due to a generic phenomenon known as critical slowing down (CSD) using descriptive statistical indicators (Wissel 1984; Scheffer et al. 2001, 2009; Carpenter et al. 2011; Lenton 2011; Chen et al. 2012; Dakos et al. 2012; Scheffer et al. 2012; but see Hastings and Wysham 2010). If a generic a phenomenon 
such as CSD exists, detection of CSD may be able to provide early warning of extinction thresholds in populations of wildlife. Recently, there has been interest in testing early warning statistical indicators of extinction thresholds in microcosm experiments (Drake and Griffen 2010; Dai et al. 2012, 2013; Veraart et al. 2012). The microcosm experiments indicate that CSD precedes extinction thresholds and can be detected using a variety of statistical indicators. However, the theory and methods have not been tested on naturally occurring populations that would be of most interest to conservation, even though populations have shown dynamics consistent with threshold crossing, such as rapid population collapse (Donald et al. 2001; Frank et al. 2005; Boettiger and Hastings 2013).

More recently, it has been shown that system-specific information can dramatically improve prediction of threshold crossings (Lade and Gross 2012). This result may be expected because the allure of detecting a generic phenomenon, such as CSD, is that a mathematical model of the dynamical system does not need to be specified (i.e., model-free detection). If an accurate model of the dynamical system is available, then in most cases, it should be straightforward to predict the threshold crossing; it is likely that the prediction from the dynamical systems model would have improved predictive skill when compared to the model-free statistical indicator approach. Furthermore, if systemspecific statistical indicators can be developed based on properties of the dynamical systems model, these too may have improved predictive skill when compared to prediction that rely solely on detecting general phenomenon (Boettiger and Hastings 2013). Populations present an optimal dynamical system to compare predictions of the fully parametric modeling approach to system-specific statistical indicators because system-specific dynamical systems models (i.e., population growth models) are well developed, as are statistical methods to estimate model parameters and associated uncertainty. In addition, using statistical properties of the population growth models, it is possible to derive statistical indicators that have theoretical justification. In this paper, we use state-space population growth models to predict a transcritical bifurcation extinction threshold in a natural population of northern bobwhite quail (Colinus virginianus) and we develop and apply theory specific to detecting a transcritical bifurcation extinction threshold in a population using a statistical indicator. Finally, we compare the predictions from the state-space population growth model to the predictions from the statistical indicator.

The paper is organized as follows: In the "Critical slowing down and the transcritical bifurcation" section, we revisit CSD and develop prerequisite theory about the transcritical bifurcation extinction threshold in populations. In the "Methods" section, we present the bobwhite quail data and the statistical methods used in our analysis and we derive a statistical indicator. In the "Results" section, we present the results of our analysis and comparison. In the "Discussion" section, we explore some technical aspects of our results and suggest areas of needed research. Finally, in the "Management implications" section, we relate the results of our study to the management of populations and the future of bobwhite quail within our study area.

\section{Critical slowing down and the transcritical bifurcation}

For simple population growth models, the dynamics responsible for CSD are easy to understand. For example, consider the Gompertz population growth model:

$N_{t+1}=N_{t} e^{r\left(1-\frac{\log \left(N_{t}\right)}{K}\right)}$

where $N_{t}$ is the current population state, $t$ is the discrete time, $r$ is the density-independent population growth rate, and $K$ is the natural $\log$ of the equilibrium population size (Gompertz 1825). For the Gompertz model, CSD occurs when $r$ approaches 0; that is, as the growth rate of a population decreases, it takes longer to return to equilibrium from an environmental perturbation (Wissel 1984). When $r$ decreases and passes through $r=0$, a transcritical bifurcation occurs and, thereafter, the population has crossed a threshold and is deterministically committed to extinction. A transcritical bifurcation is a type of bifurcation in which equilibrium points exchange stability as a parameter is changed (Strogatz 1994). A feature common to many population growth models is that a change in the densityindependent growth rate $(r)$ from positive to negative results in a qualitative change to the dynamical regime. When $r>0$, there is a stable equilibrium point for $N>0$ and an unstable equilibrium point at $N=0$ (i.e., extinction), whereas when $r<0, N=0$ becomes stable and $N>0$ becomes unstable. If a transcritical bifurcation adequately describes an extinction threshold, the discovery could have profound implications for the management and conservation of fisheries and wildlife because the extinction threshold could be crossed prior to the population showing traditional warning signs of extinction (e.g., population decline). The CSD that precedes the transcritical bifurcation is a phenomenon common to many continuous and discrete time stochastic population models (e.g., Beverton-Holt, logistic, Ricker, theta-logistic) and detecting CSD can be used to warn of the transcritical bifurcation extinction threshold (Drake and Griffen 2010).

\section{Methods}

Bobwhite quail data

The northern bobwhite quail (C. virginianus) is an ideal study organism to determine if an impending extinction 
threshold can be detected in field data because populations have declined during recent decades ( $\sim 3.8 \%$ annually; Sauer et al. 2011), likely due to habitat deterioration and loss (Roseberry et al. 1979; Veech 2006). This slow anthropogenic forcing may be analogous to the deteriorating environmental conditions of microcosm experiments demonstrating the transcritical bifurcation (Drake and Griffen 2010).

Whistle count (WC) data were collected by the Nebraska Game and Park Commission from 1965 to 2011 and consisted of four roadside survey routes in the tall grass prairie eco-region in the extreme southeastern portion of Nebraska. Each route was located entirely in one of four Nebraska counties: Johnson, Nemaha, Otoe, and Richardson. Each survey route consisted of a single biologist starting at a fixed point in the easternmost portion of the survey area and recording the number of unique whistling males heard in $2 \mathrm{~min}$. The biologist then moved west approximately $1 \mathrm{mi}$ and repeated the process until 20 point counts were obtained. Stops did not occur near farm yards. The surveys were conducted between 15 June and 10 July, beginning at sunrise. Surveys were conducted only if air temperature at the first stop was $<21.1{ }^{\circ} \mathrm{C}$, if it was not raining, and if winds speeds were $<19.3 \mathrm{~km} \mathrm{~h}^{-1}$. If a survey was initiated, but wind speeds were $>19.3 \mathrm{~km} \mathrm{~h}^{-1}$ and/or if it was raining at a stop, the count was recorded as not available. The WC route averages were available for all years of the study. Historically, route averages were the only data archived and counts at each route stop are unavailable for the entire study period. Route averages were calculated by summing the total number of unique whistling males heard at each stop and dividing by the number of survey stops with available counts. Since the number of total stops was variable due to the sampling protocol, we multiplied the route average by 20 and rounded to the nearest integer to obtain the population index used for our analysis. Judging by more recent data, it appears that route averages were most often calculated from the full 20 stops. It appears the biologists who collected the WC data took great caution to initiate surveys only when it was anticipated that the full 20 stops could be obtained; therefore, we expect that the population index used in our analysis is the true route total for most observations.

For comparison purposes, we used an independent data source with a different sampling protocol, the Breeding Bird Survey (BBS) data; we used raw count route totals from 1967 to 2011 for Nebraska routes 1-3, which were conducted in the extreme southeastern Nebraska counties of Gage, Johnson, Nemaha, Pawnee, and Otoe (USGS Patuxent Wildlife Research Center 2012). The BBS data included several missing counts, and in total, $18.5 \%$ of the data was missing. If the analysis of the BBS data corroborates the results of the WC data analysis, we will have stronger support for our conclusions. In addition, the BBS data is widely available, but of lower quality (i.e., less strict protocols and more missing data) than the WC data; detection of the transcritical bifurcation in the BBS data is a test of our methods with data that is available for other species and study areas.

State-space population models

Threshold crossing and transitions to alternative states in populations have been well documented and described by dynamical systems (Holling 1973; Bascompte 2003; Ives et al. 2008; Schooler et al. 2011). Dynamical systems models, however, have not been used to detect the transcritical bifurcation extinction thresholds in natural or microcosm populations (Drake and Griffen 2010). The lack of application in natural populations may be a result of the complex statistical methods that must be used to adequately model nonlinear population dynamics and observational error. When observational ecological data is available, statespace time series analysis methods have allowed researchers to model the data collection process along with realistic dynamical systems models capable of threshold dynamics (Ives et al. 2003, 2008; Schooler et al. 2011). Methods to fit state-space population growth models to observational time series data are well developed and it would be straightforward to estimate model parameters and determine if the growth rate has decreased and if an extinction threshold has or will be crossed (de Valpine and Hastings 2002; Williams et al. 2003; Clark and Bjørnstad 2004; Dennis et al. 2006; Wang 2007; Pedersen et al. 2011; Nadeem and Lele 2012).

We used a multivariate state-space Gompertz model that was fit simultaneously to all routes for each data set. The form of the model was:

$$
\begin{aligned}
& N_{i, t+1}=N_{i, t} e^{r_{t}\left(1-\frac{\log \left(N_{i, t}\right)}{K_{t}}\right)+\varepsilon_{i, t}} \\
& \boldsymbol{\varepsilon}_{t} \sim \operatorname{MVN}\left(\mathbf{0}, \sum=\sigma_{p}^{2} \boldsymbol{I}_{n}+\rho_{p} \sigma_{p}^{2}\left(\boldsymbol{I}_{n}-\boldsymbol{J}_{n}\right)\right)
\end{aligned}
$$

$Y_{i, t} \sim \operatorname{Poisson}\left(N_{i, t}\right)$

where $N_{i, t}$ is the population state of the $i$ th route (of $n$ total routes) at time $t$. The time-dependent growth rate is $r_{t}=-$ $r_{\max }+r_{\Delta} t>0$ where $r_{\max }$ is the density-independent maximum growth rate (assuming $r_{\Delta}$ is negative) and $r_{\Delta}$ is the linear trend in $r_{t}$. The natural $\operatorname{logarithm}(\log )$ of the equilibrium population size at time $t$ is $K_{t}=K_{\max }+K_{\Delta} t>0$, where $K_{\max }$ is the maximum equilibrium population size (assuming $K_{\Delta}$ is negative) and $K_{\Delta}$ is the time-dependent trend in $K_{t}$. The observed population size $\left(Y_{i, t}\right)$ is described by a Poisson distribution. The process error term $\varepsilon_{t}$ is distributed multivariate normal with a mean of 0 and compound symmetry 
variance-covariance matrix, where $\sigma_{p}^{2}$ is the environmental process variance and $\rho_{p}$ is the correlation in process error among routes within a year. The $\rho_{p}$ models the spatial correlation of process error. We also fit a multivariate state-space formulation of the Ricker (1954) model:

$N_{i, t+1}=N_{i, t} e^{r_{t}\left(1-\frac{N_{i, t}}{K_{t}}\right)+\varepsilon_{i, t}}$

All model parameters are the same as the Gompertz model, except that $K_{t}$ is the time-dependent equilibrium population size, rather than the natural logarithm of equilibrium population size.

First-order nonlinear difference equations, such as the Gompertz or Ricker model, were chosen a priori to describe the population dynamics of bobwhite quail based on life history traits of the species and limitations of the data. The WC and BBS data were population-level data and lacked the detail to develop mechanistic models based on individual characteristics such as matrix projection models or integral protection models (Easterling et al. 2000; Caswell 2001; Lebreton and Gimenez 2013). Therefore, we limited our methods to fitting phenomenological models that describe dynamics at the population level. We chose to use discrete time difference equations because bobwhite quail generally produce one brood annually and annual survival of adults is low (Roseberry and Klimstra 1984; Hastings 1996). In addition, the WC and BBS data were collected annually. Therefore, a difference equation with annual time steps is appropriate to model the population dynamics of the bobwhite quail and the data collection process.

A challenge when using nonlinear difference equations to model population dynamics is determining the functional form of density dependence (Williams 2013). For example, the Ricker model assumes that the realized growth rate declines linearly as population size increases, whereas the Gompertz model assumes that the realized growth rate declines linearly as the natural logarithm of population size increases. A priori, we would have chosen the theta-logistic population growth model. The theta-logistic population growth model is flexible in describing the functional form of density dependence. However, it is well known that the theta-logistic model suffers from weakly or unidentifiable parameters, and this was the case in our study (see the "Discussion" section; Polansky et al. 2009; Clark et al. 2010). Therefore, we were required to choose the functional form of density dependence and compare functional forms using graphical model checks and model selection methods. We chose the density-dependent relationship of the Gompertz and Ricker models a priori based on the life history traits of the bobwhite quail and by graphical examination of the observed growth rate $\left(r_{\text {observed }, i, t}=\log \left(\frac{Y_{i, t+1}}{Y_{i, t}}\right)\right)$ plotted against the observed population size $\left(Y_{i, t}\right)$ for the WC and BBS data. The bobwhite quail is characterized as an $r$ selected species and is known to have a high reproductive capacity and low annual survival (Roseberry and Klimstra 1984). Life history strategies can impact the functional form of density dependence, and it has been suggested that $r$ selected species are theoretically expected to exhibit a pattern of strong reduction in the observed growth rate at small population sizes because of larger consumption of resources by the increased reproductive output (Williams 2013). This would suggest that the Gompertz model may be the a priori best model to fit to the data. In addition, the plots of $r_{\text {observed, } i, t}$ against the observed population size $\left(Y_{i, t}\right)$ suggested that the observed growth rate is affected by strong density dependence at small population sizes. We chose the Ricker model because it can be difficult to determine the functional form of density dependence and we wanted to allow for comparison with other forms, so that we can potentially support or refute our a priori model justification using statistical model selection techniques.

Parameter estimation for state-space population models is well developed using maximum likelihood (ML) or posterior sampling under a Bayesian paradigm (de Valpine and Hastings 2002; Clark and Bjørnstad 2004; Dennis et al. 2006; Wang 2007; Ponciano et al. 2009; Pedersen et al. 2011; Nadeem and Lele 2012). For our situation, we feel that ML estimation is desirable because our results could be sensitive to the specification of vague priors. A sensitivity analysis to justify our conclusions would be cumbersome. We used the data cloning (DC) algorithm to obtain ML parameter estimates (Lele et al. 2007, 2010; Ponciano et al. 2009; Nadeem and Lele 2012). The DC algorithm uses Markov chain Monte Carlo (MCMC) posterior sampling to obtain ML parameter estimates and the associated asymptotic variance-covariance matrix. Using DC methods can be a bit more involved than MCMC sampling under a Bayesian paradigm. Most notably, the number of data clones $(c)$ must be increased until the posterior distribution of the model parameters is nearly degenerate. This is shown numerically when the standardized largest eigenvalue of the parameter variance-covariance matrix $\left(\lambda_{s}\right)$ approaches 0 at the rate $\frac{1}{c}$ (Lele et al. 2010). A predetermined cutoff value of $\lambda_{s}$ is specified and, when reached, the DC algorithm is assumed to have converged. In addition, convergence of the MCMC algorithm must also be monitored. Although this may appear cumbersome, fairly automated software has been developed to complete the task (Sólymos 2010). Furthermore, when the DC algorithm has converged, all model parameters have been shown numerically to be identifiable (Lele et al. 2010). With the large number of parameters in our nonlinear population growth models, parameter identifiability may be questionable; the DC algorithm can numerically demonstrate that model parameters are identifiable (Lele et al. 2010). For our analysis, 
we assumed that the DC algorithm had converged when $\lambda_{s}<0.05$ and appeared to visually decrease at the rate $\frac{1}{c}$ (Sólymos 2010). We also assumed that the MCMC simulation had converged when the multivariate scale reduction factor was $<1.1$ (Brooks and Gelman 1998). When $\lambda_{s}$ did not decrease at the rate $\frac{1}{c}$, the unidentifiable parameter was identified using diagnostic plots and removed from the model, and parameters were estimated for the reduced model (Lele et al. 2010).

All quantities derived from the population models (e.g., population prediction intervals and distributions of statistical indicators) and confidence intervals (CI) were obtained by bootstrap integration over the asymptotic distribution of the model parameters using 50,000 bootstrap samples (Nadeem and Lele 2012). For these bootstrap simulations, we assumed that $r_{t} \geq 0, K_{t} \geq 0.01$, and $N_{t} \geq 1$ for all $t$. These restrictions were required to avoid numerical issues associated with the population models postbifurcation. CIs for all other derived quantities were obtained from the equal tail percentiles of one million parametric bootstrap samples (Efron and Tibshirani 1993). When the derived quantities were linear combinations of model parameters, we obtained standard errors from linear transformations of the asymptotic variancecovariance matrix and constructed Wald-type CIs (Ponciano et al. 2009). Our methods were well documented and described by Nadeem and Lele (2012), with the only difference being that we are fitting multivariate populations models and allowing population model parameters to have time-dependent trends.

We compared the Ricker and Gompertz models using Akaike's information criterion adjusted for small sample size $\left(\mathrm{AIC}_{\mathrm{c}}\right.$; Burnham and Anderson 2002). Although likelihood values cannot be easily calculated for our state-space population models, calculating the likelihood ratio is relatively straightforward and, therefore, calculating the difference in $\mathrm{AIC}_{\mathrm{c}}\left(\Delta \mathrm{AIC} \mathrm{C}_{\mathrm{c}}\right)$ between the two models is feasible. Our goal in fitting multiple population models and comparison using $\mathrm{AIC}_{\mathrm{c}}$ was not necessarily consistent with the goals of model selection and multimodal inference (Burnham and Anderson 2002). Instead, we intended to find population models that graphically described the observed time series and statistical indicator well. Our approach to model comparison and criticism is similar to posterior predictive checks under a Bayesian paradigm (Gelman et al. 2004); however, instead of comparing the posterior distribution with the observed data and statistical indicators, we compare the bootstrap distributions. We used the program $\mathrm{R}$ (version 2.15.1) for all statistical computing ( $\mathrm{R}$ Project for Statistical Computing, Vienna, Austria). We used JAGS (version 3.2.0) for all MCMC sampling (Plummer 2012) called from the dclone package (version 1.8-1) in program R (Sólymos 2010).
Statistical indicator

Population variability can be driven by the interaction between environmental variability (density-independent process error) and density dependence (Williams et al. 2003). The population variability driven by environmental variability should be amplified by a weakening growth rate prior to the transcritical bifurcation (Drake and Griffen 2010). This is analogous to what others have termed CSD, where, due to a reduced growth rate, the population returns to equilibrium from perturbations slower as the threshold is approached (Drake and Griffen 2010; Scheffer et al. 2012). If this amplification in population variability is detectable, then the extinction threshold may be anticipated.

We propose the statistical indicator SVL, which is the annual between survey-route sample variance of the natural log-transformed population counts. The Gompertz model provides analytically tractable justification for a statistical indicator. It can be shown (see the Appendix) for the Gompertz model in Eq. 2 that:

$$
\begin{aligned}
& \operatorname{Var}\left(\log \left(N_{t}\right)\right) \\
& =\frac{\sigma_{p}^{2}\left(1-\rho_{p}\right)}{2\left(\frac{r_{t}}{K_{t}}\right)-\left(\frac{r_{t}}{K_{t}}\right)^{2}}\left(1-\left(1-\frac{r_{t}}{K_{t}}\right)^{2 t}\right) \approx \frac{\sigma_{p}^{2}\left(1-\rho_{p}\right)}{2\left(\frac{r_{t}}{K_{t}}\right)-\left(\frac{r_{t}}{K_{t}}\right)^{2}} .
\end{aligned}
$$

The main result is that the theoretical variance of the logtransformed population state depends only on the process error and correlation, the time $(t)$ since initiation (i.e., the stationarity of the distribution), the growth rate, and the equilibrium population size. For reasonable $t(t>1)$ and values of $\frac{r_{t}}{K_{t}}$, the theoretical variance is approximately equal to a convex function that depends only on $r_{t}, K_{t}$, and $\sigma_{p}^{2}(1-$ $\left.\rho_{p}\right)$. As $r_{t}$ approaches 0 , the theoretical variance increases nonlinearly to infinity along a vertical asymptote and $\lim _{r_{t \rightarrow 0}} \frac{\sigma_{p}^{2}\left(1-\rho_{p}\right)}{2\left(\frac{r_{t}}{K_{t}}\right)-\left(\frac{r_{t}}{K_{t}}\right)^{2}}=\infty$ suggests that estimating when the transcritical bifurcation occurs is equivalent to determining the location of the vertical asymptote along the time axis. Estimating the location of the vertical asymptote along the time axis can be used to predict when the transcritical bifurcation will occur. Since the asymptotic distribution of $\log \left(N_{\infty}\right)$ is approximately normally distributed (Lande et al. 2003), an estimator of $\operatorname{Var}\left(\log \left(N_{t}\right)\right)$ is the annual between-survey-route sample variance of the log-transformed population state:

$\mathrm{SVL}_{t}=\frac{1}{n-1} \sum_{1}^{n}\left(\log \left(N_{i, t}\right)-\overline{\log \left(N_{t}\right)}\right)^{2}$

where $N_{i, t}$ is the population state of the $i$ th route (of $n$ total routes) at time $t$. However, since the observation 
process results in measurement errors, we suggest substituting the observed annual route count totals for the population state $\left(N_{i, t}\right)$ in Eq. 6. Although the theoretical variance for the Poisson log-normal state-space formulation of the Gompertz model (i.e., Eqs. 2 and 3), which accounts for observational error, becomes analytically intractable, it is easy to show by numerical simulation that SVL increases nonlinearly to infinity along a vertical asymptote as $r_{t}$ goes to 0 as expected from the analytical results which do not account for observer error (see the results from Fig. 3). This suggests that the statistical indicator SVL is robust to observational error. Furthermore, this phenomenon appears to be generic among many population models, including the Ricker model. To show this in the analytically intractable case of observational error and for models other than the Gompertz model, we plot the bootstrap distribution of SVL simulated from the state-space models with model parameters estimated from the WC and BBS data (Fig. 3).

To determine if SVL is a useful indicator, we compare the observed time series of SVL (Eq. 6) calculated from the WC and BBS data to the bootstrap distribution from the statespace population models. Comparing the observed SVL to the bootstrap distribution allows us to determine if the observed SVL follows the theoretically expected relationship. To empirically detect the transcritical bifurcation, we fit a generalized nonlinear model (GNLM) with a gamma response distribution to the time series of SVL. GNLMs are similar to generalized linear models, except that, instead of specifying a link function and a linear predictor (Stroup 2012), which is linear in the model parameters, we specify a mean function, which can be nonlinear with respect to the model parameters. We fit the gamma GNLM using the mean function:

$\mu_{t}=\frac{\beta_{1}}{2\left(\beta_{2} t\right)-\left(\beta_{2} t\right)^{2}}$

where $\beta_{1}$ and $\beta_{2}$ are the model parameters and $t$ is the time in years. Comparing Eq. 7 to Eq. 5 , it is clear that $\beta_{1}$ is an estimate of $\sigma_{p}^{2}\left(1-\rho_{p}\right)$ and $\beta_{2} t$ is an estimate of $\frac{r_{t}}{K_{t}}$, hence $\mu_{t}$ is an estimate of $\operatorname{Var}\left(\log \left(N_{t}\right)\right)$. GNLMs are an easily accessible phenomenological method to detect changes in the observed SVL. The gamma distribution is a natural choice to model variances, and the mean function $\left(\mu_{t}\right)$ has theoretical justification and appears to be a good description of the expected nonlinear increase in SVL. Estimating the date when the transcritical bifurcation is expected to occur can be accomplished by determining the year $(t)$ when the denominator of Eq. 7 is equal to 0 (i.e., the year $(t)$ when $\widehat{\mu}_{t}=\infty$ ) and the $95 \%$ CI for this date can be calculated using parametric bootstrapping. This date corresponds to the estimated date when $r_{t}=0$ and occurs when SVL is predicted to become infinite. Because the gamma distribution is undefined for an
SVL of 0 (i.e., all survey total route counts for the year are the same), we added a small ( 0.01$)$ positive constant to SVL estimates of 0 . When that survey total route count was zero (i.e., $Y_{i, t}=0$ ), SVL was undefined, so we added 1 to the count. An observed count of 0 occurred twice in the BBS survey data and did not occur in the WC data. For the BBS data, when only one route was available in a given year, we did not calculate SVL and assumed that it was missing.

\section{Results}

The population growth rate $\left(r_{t}\right)$ decreased during the survey period. Although the exact estimate of $r_{t}$ depended on the assumed population growth model and data set, the decreasing trend was ubiquitous for all population models in both WC and BBS data (Fig. 1). Using the WC data, the estimated date when the transcritical bifurcation occurred (i.e., when $\left.r_{t}=0\right)$ was $2010(2002-2036 ; 95 \% \mathrm{CI})$, assuming the Gompertz model, and 2007 (1999-2065; 95 \% CI), assuming the Ricker model. Similarly, using the BBS data, the estimated date when the transcritical bifurcation occurred was 2008 (1999-2064; $95 \%$ CI), assuming the Gompertz

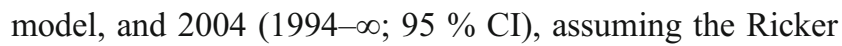
model. The equilibrium population size $\left(e^{K_{t}}\right)$ showed a decreasing trend over the study period for both WC and BBS data when the Gompertz model was assumed; however, this trend was not statistically significant, as evident by the increasing upper $95 \%$ CI (Fig. 2). Estimated equilibrium population size, assuming the Gompertz model, on the date of bifurcation was $37.4(13.6-90.0 ; 95 \% \mathrm{CI})$ using the WC data and $11.9(3.2-43.8 ; 95 \% \mathrm{CI})$ using the BBS data (Fig. 2). We were unable to fit the Ricker model that allowed for a decreasing trend in $K_{t}$ because $K_{\Delta}$ was unidentifiable in both the WC and BBS data sets. Therefore, the equilibrium population size $\left(K_{t}\right)$ as estimated by the Ricker model was constant through the study period and was estimated to be $61.2(45.0-77.4 ; 95 \% \mathrm{CI})$ using the WC data and 32.9 (22.0-43.7; $95 \%$ CI) using the BBS data (Fig. 2). Similarly, the observed count $\left(Y_{i, t}\right)$ and estimated observed count $\left(\widehat{Y_{i, t}}\right)$ was also relatively large (Fig. 3, inset plots). Equilibrium population sizes and observed counts much greater than 0 suggest that neither small nor decreasing population size will warn of the transcritical bifurcation extinction threshold.

The observed SVL from both WC and BBS data showed a similar nonlinear increasing trend. The pattern in the observed SVL was well explained by the bootstrap distribution of SVL estimated from the Gompertz model as evident from the estimated expected trend and $95 \%$ CIs from the bootstrap distribution of SVL (Fig. 3). The Ricker model showed a similar pattern in SVL, but does not describe the observed SVL as well as the Gompertz model as judged by the $95 \%$ 
Fig. 1 Estimated timedependent population growth rate $\left(r_{t}\right)$ with $95 \%$ CIs (dashed lines) assuming the Gompertz and Ricker population growth models using WC survey data and BBS data for northern bobwhite quail in southeastern Nebraska. The vertical gray lines are the estimated date of the transcritical bifurcation (solid lines) and associated $95 \%$ CIs (dashed lines)
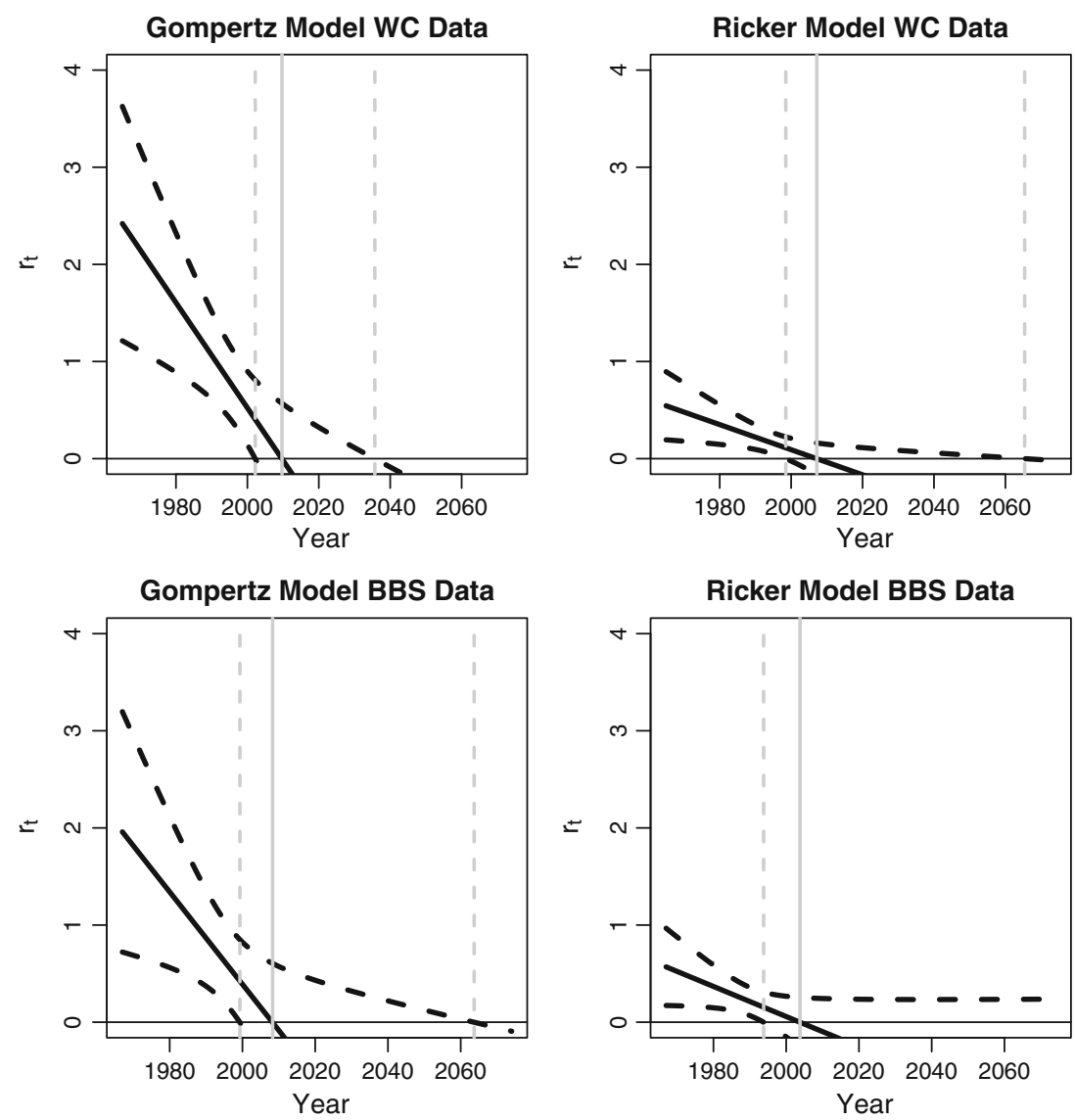

bootstrap CIs. Regardless, both population models and data sets confirm that SVL was increasing as $r_{\mathrm{t}}$ decreased and the transcritical bifurcation was approached.

Using our GNLM methods, which rely only on the observed SVL, we estimated that the transcritical bifurcation will occur in 2018 (2004-2031; $95 \%$ CI) using the WC data and will occur in 2012 (2006-2018; $95 \%$ CI) using the BBS data. The SVL provides similar predictions as our statespace population growth model as the CIs are broadly overlapping. In addition, the mean function and associated $95 \%$ CIs describing the observed SVL are similar to the mean function and $95 \%$ CIs of SVL based on the bootstrap distribution from the Gompertz and Ricker models. It appears that our GNLM methods provide a reasonable model describing the observed increase in SVL.

Population prediction intervals based on the Gompertz and Ricker models appear to explain the observed time series survey data well (Fig. 3 inset). Based on these plots alone, it would be difficult to declare which model best described the data, although the deterministic declining trajectory of the Gompertz model appears to fit the observed data better. The observed SVL compared to the bootstrap distribution of SVL from the population models suggests that both the Gompertz and Ricker models explain the increasing trend. The $\Delta \mathrm{AIC}_{\mathrm{c}}$ between the Ricker and Gompertz models was 1.2 for the WC data and 1.9 for the BBS data. Again, neither model appears to excessively outperform the other with respect to both data sets; however, the Gompertz model does appear graphically to fit the data better than the Ricker model and this slight advantage is also supported by the $\Delta \mathrm{AIC}_{\mathrm{c}}$ values.

\section{Discussion}

Our results are the first to detect a transcritical bifurcation extinction threshold using state-space population models and field data collected from a wildlife population. Our results indicate that the population of bobwhite quail in the extreme southeastern portion of Nebraska has crossed or will cross, in the near future, the transcritical bifurcation extinction threshold. This conclusion is well supported by two independent data sets and model-based inference based on ML. An initial assumption might have been that the dynamics of a population driven to extinction would result in the equilibrium population size decreasing to 0 and the growth rate remaining constant (Huang et al. 2012). If this were the case, the population would track the declining equilibrium population size and declining population size should warn of extinction. As our results show, the growth rate can decrease over time. When the growth rate becomes negative, the population will be committed to extinction. Our results show that the growth rate becomes negative long before extinction 
Fig. 2 Estimated time dependent equilibrium population size with $95 \%$ CIs (dashed lines) assuming the

Gompertz $\left(e^{K_{t}}\right)$ and Ricker $\left(K_{t}\right)$ population growth models using WC survey data and BBS data for bobwhite quail in southeastern Nebraska
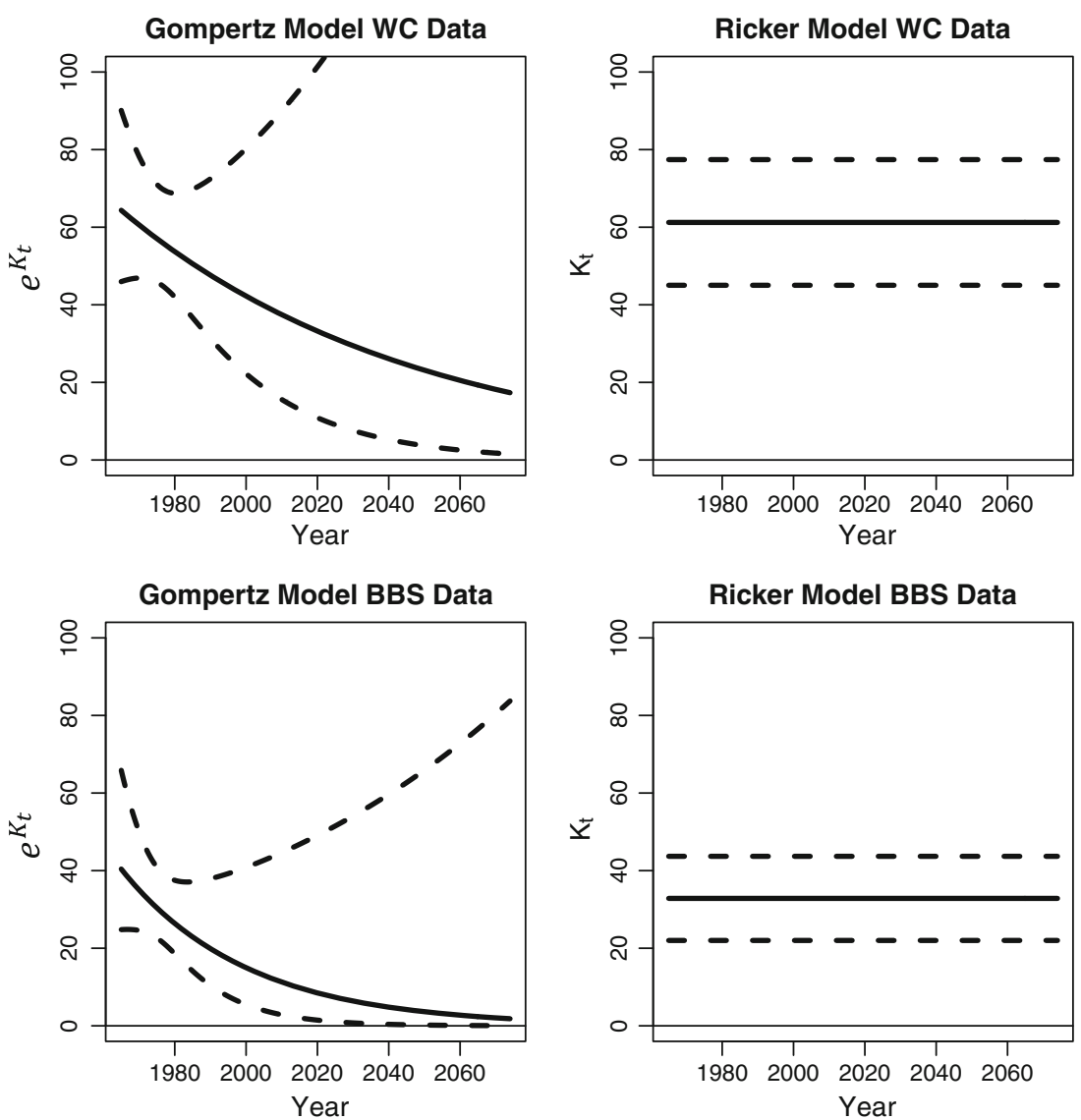

due to a decreasing equilibrium population size. These dynamics result in an extinction threshold, marked by a transcritical bifurcation, that standard methods such as population trend analysis or population viability analysis assuming population growth models with constant parameters would not detect (Nadeem and Lele 2012).

Similarly, our results are the first to detect a transcritical bifurcation with a statistical indicator using field data collected from a wildlife population. Others have suggested that statistical indicators may fail in situations when observational errors are large (Dai et al. 2012; Carpenter et al. 2011; Ives and Dakos 2012). In both WC and BBS data, observational errors are significant, yet predictions based on the statistical indicator were similar to our state-space model which explicitly accounted for observation error (de Valpine and Hastings 2002; Nadeem and Lele 2012). Based on numerical simulations and corroborating predictions from state-space models, it appears that the statistical indicator SVL is robust to observational error. Furthermore, our theoretical results suggest a critical level of SVL that allows us to predict when the transcritical bifurcation extinction threshold will be crossed without a reference population and allows for statistical inference using GNLMs. Lack of inference procedures and critical levels of statistical indicators has been a major limitation even when a reference system is available (Drake and Griffen 2010; Carpenter et al. 2011; Dai et al. 2012; Ives and Dakos 2012; Boettiger and Hastings 2013). Our methods based on SVL overcome these limitations. However, we feel that comparisons of state-space models, SVL, and other generalized model-based approaches developed to detect thresholds would be useful (Wissel 1984; Ives and Dakos 2012; Boettiger and Hastings 2012a, b; Lade and Gross 2012).

Alternative explanations for the observed increase in SVL could be decreasing equilibrium population size, increased environmental variability (Williams et al. 2003), various forms of demographic stochasticity (Melbourne and Hastings 2008), or alternative thresholds. The theoretical SVL based on specific population models is likely a complex function of equilibrium population size, population growth rate, and environmental stochasticity. For the Gompertz model, the relationship between equilibrium population size and SVL is complex, and for most other population models, the relationship can only be explored numerically. Our numerical simulations of SVL (Fig. 3) seem consistent with SVL increasing due to a decreasing growth rate, in that if we set $K_{\Delta}$ to 0 for the Gompertz model, the increasing trend in SVL is still present. If nonstationary environmental variability is a plausible explanation of the observed increase in SVL, process error $\left(\sigma_{p}^{2}\right)$ would have had to trend similarly to the nonlinear increasing pattern in SVL. Nonlinearly increasing process error due to increasing environmental variability seems like an improbable explanation given that 

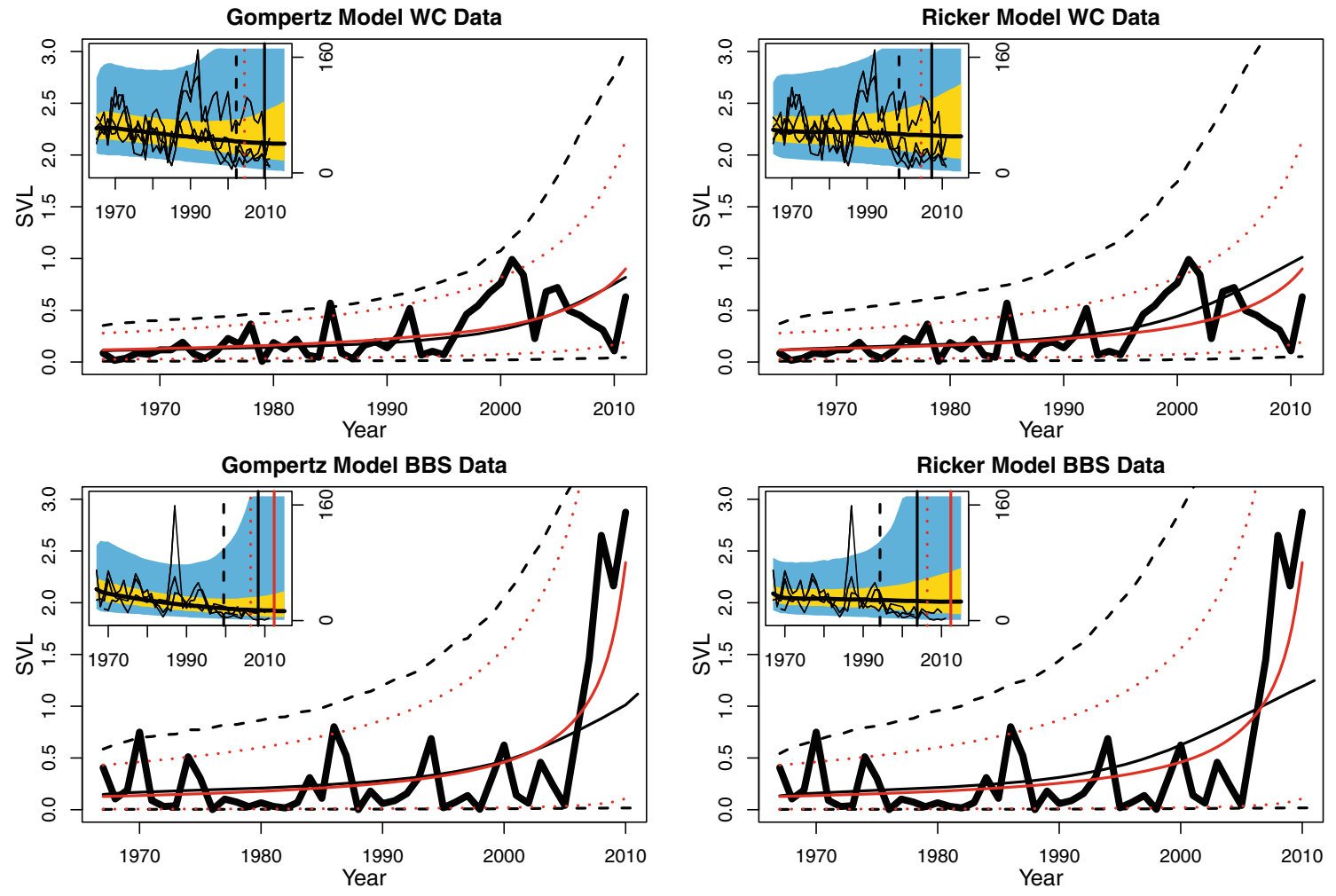

Fig. 3 Statistical indicator SVL (thick black line; Eq. 6) estimated from the bobwhite population using WC data and BBS data with mean (thin black line) and $95 \%$ CI (black dashed line) from the bootstrap distribution of SVL estimated from the Gompertz or Ricker state-space population models. The red line is the mean function (Eq. 7) and $95 \% \mathrm{CI}$ (dotted red line) from a GNLM used to describe the increasing trend in the statistical indicator SVL. The inset plot contains population time series data (thin black lines) along with the expected population

our theory, supported by a decreasing growth rate, matches the empirical data well. If any form of demographic stochasticity was substantially influencing SVL, we would expect SVL to increase in the population as the population was declining. We did not test this hypothesis in our example, but if the population has declined to the point that demographic stochasticity influences SVL, it seems likely that conservation measures should be taken. In this case, the potential loss due to false detection of the transcritical bifurcation seems minimal. In addition to the transcritical bifurcation extinction threshold identified in our state-space model, other mechanisms in the population dynamics may exist that result in threshold dynamics, such as an Allee effect (Hoffman et al. 2010; Dai et al. 2012; Lade and Gross 2012). Although we cannot rule out that SVL is detecting alternative extinction thresholds, the generality of CSD suggests that we might expect the same patterns in SVL, regardless of the mechanism generating the threshold (Wissel 1984).

It could be argued that we should have fit a wider array of population models (e.g., theta-logistic) and reduced (e.g., Gompertz with $r_{\Delta}=0$ ) and expanded (e.g., $r_{t}=r_{\max }+r_{\Delta} t+r_{2} \Delta t^{2}$, where $r_{2 \Delta}$ is the coefficient of the second-order polynomial

count (thick black line) and $50 \%$ (gold) and $95 \%$ (blue) CIs under the assumed population model. The vertical black line is the estimated date of the transcritical bifurcation along with the lower $95 \%$ CI (black dashed line) based on the assumed population model. The vertical red line is the estimated date of the transcritical bifurcation with the lower $95 \%$ CI (red dashed line) that was estimated with the GNLM using the statistical indicator SVL

time-dependent change in $r_{t}$ ) population growth models and observational error distribution (e.g., overdispersed Poisson; Knape et al. 2011). We then could compare several plausible explanations of our data using our graphical comparison and $\mathrm{AIC}_{\mathrm{c}}$. Such a comparison would be ideal; however, it was not feasible for our study. For example, we fit a state-space formulation of the theta-logistic, but our estimation procedure failed unless we specified the shape parameter which determined the functional form of density dependence. Potential difficulties when fitting the theta-logistic are well known (Polansky et al. 2009; Clark et al. 2010), and failure of our estimation procedure could be attributed to unidentifiable parameters. Still, we could have expanded our model set by including fewer parameters in the Gompertz and Ricker models. We in fact did do this (e.g., state-space Gompertz with $r_{\Delta}=0$ and $K_{\Delta}=0$ ), but this approach was unproductive because there were some obvious features common to both data sets that we feel a population model must explain, such as the decreasing trend in abundance and increasing trend in SVL. The parameterizations of the state-space models (SSMs) in our analysis appear to be the simplest parameterizations that adequately describe these dynamics. Furthermore, we could 
have fit models that allowed for polynomial trends in $r_{t}$ and $K_{t}$. We explored the potential of this, but found that MCMC sampling was difficult due to highly correlated parameters; again, this is likely due to unidentifiable parameters. We did not evaluate alternative observational error distributions for our SSM. It is unlikely that our data could identify the correct observation error model because we lacked replication at the route level (Knape et al. 2011). We attempted to fit some alternative distributions to describe observational error (e.g., log-normal), but it appeared that the error component parameters were weakly identifiable. A more serious concern regarding SSM observation error model is nondetection. Methods to correct for nondetection in SSMs require replicated counts or additional continuous covariates that affect detection (Hefley et al. 2013). Obtaining useful covariates that affect detection (e.g., wind speed) would be difficult or impossible for all years of our study. We do not expect the predicted dates of the bifurcation to be highly sensitive to specification of the observation error model, although exact population model parameter estimates may vary.

Additionally, we could have considered covariates that may potentially explain the trends in $r_{t}$ and $K_{t}$. Habitat deterioration and loss are difficult to quantify, and accessible data (e.g., National Land Use Dataset) do not have the temporal resolution required for our study. Showing the dependence of population growth model parameters on habitat covariates is an area of needed research (Griffen and Drake 2008) because, although we can phenomenologically model the effects of covariates by assuming that model parameters change linearly with time, it would be highly desirable to know which habitat variables are potentially influencing the population so that remedial actions can be taken.

Even if we had expanded our model set, we question the utility of $\mathrm{AIC}_{\mathrm{c}}$ in our situation. Variants of the AIC are well established for model selection and have been used for comparison of SSMs (Burnham and Anderson 2002; de Valpine and Hastings 2002; Nadeem and Lele 2012). The utility of model selection tools for threshold detection is a needed area of research if SSMs are to be used for detecting the transcritical bifurcation. The $\mathrm{AIC}_{\mathrm{c}}$ used in our analysis does not take parameter uncertainty into consideration. Calculating the marginal likelihood for SSMs requires integrating out the random population state. The population state can be highly influenced by the timing of the bifurcation. When using $\mathrm{AIC}_{\mathrm{c}}$, the uncertainty in the model parameters used to estimate the bifurcation date is not taken into account and we suspect that doing so may influence model selection. Secondly, post-bifurcation, the stochastic Gompertz and Ricker models are no longer valid population models as initially defined. Post-bifurcation population dynamics are unknown and both stochastic population models may result in implausible dynamics. For example, after the transcritical bifurcation, the population state could diverge to infinity depending on the value of the population state prior to the bifurcation. A biologically plausible model would converge to zero. We suspect that post-bifurcation population dynamics will likely influence parameter estimation and model selection. For our results, it appears that the estimated population model parameters are plausible, but we are unsure of $\mathrm{AIC}_{\mathrm{c}}$-based model selection results. Since time series data are relatively easy to visualize, at present, we prefer our graphical model criticism approach.

\section{Management implications}

Our results suggest that the population of bobwhite quail in the extreme southeastern portion of Nebraska has crossed the transcritical bifurcation extinction threshold or will in the near future. We expect extinction soon after, but due to bifurcation delay, there will be an unknown amount of time between when the threshold is crossed and when extinction occurs (Kuehn 2011). Drake and Griffen (2010) experimentally induced a transcritical bifurcation in populations of Daphnia magna. In this experiment, habitat deterioration caused by a reduction in food started on day 154 and the transcritical bifurcation occurred on day 271, with the mean date of extinction on day 297 ( $\mathrm{SE}=16.4$ days). The amount of time between transcritical bifurcation and extinction is undoubtedly dependent on the species and environmental process driving extinction.

The drivers of the extinction threshold in the bobwhite population are unknown. We speculate that a slow anthropogenic forcing due to habitat deterioration and loss caused the density-independent growth rate to decrease. In our study area, habitat deterioration likely occurred due to a decrease in crop diversity (Hiller et al. 2009) and a decrease in the abundance of weedy species within agricultural fields as a result of genetically modified herbicide-tolerant crops (Watkinson et al. 2000). Habitat loss may have also occurred as a result of increased agricultural field size and decrease in brushy fencerow habitat, both of which have been documented in our study area (Baltensperger 1987; Hiller et al. 2009). Recovery of populations that have crossed the transcritical bifurcation extinction threshold has not been shown in microcosm or natural populations. We expect that microcosm experiments will provide the first insights into the reversibility of the transcritical bifurcation.

The result that the equilibrium population sizes and observed abundance of the bobwhite quail population was much greater than 0 on the date that the extinction threshold was estimated to be crossed suggests that neither small nor decreasing population size will warn of the transcritical bifurcation extinction threshold. Managers of populations in regions experiencing land use changes need to consider the implications of this result. Early detection of extinction 
thresholds is essential to ensure the persistence of species when habitat changes over time (Krebs et al. 1999; Donald et al. 2001; Hole et al. 2002; Green et al. 2005; Ringsby et al. 2006; Biggs et al. 2009). This is especially true in situations where the land use change is relatively slow because the extinction thresholds may be detectable long before the threshold is crossed, but once crossed, the land use changes may be slow, difficult, and potentially impossible to reverse, even if the population could be recovered.

Acknowledgments We thank the Nebraska Game and Parks Commission for collecting the whistle count data. Data collection efforts were supported by the Federal Aid in Wildlife Restoration Project W-15-R-68. Requests for data should be addressed to Nebraska Game and Parks Commission Upland Game Program Manager (jeff.lusk@nebraska.gov). We thank all the volunteers who participated in the Breeding Bird Survey. We thank the editor, two anonymous reviewers, S. Fritz, M. Sliwinski, B. Tenhumberg, and the Eco Chat group, particularly C. Brassil and B. Nolting, for comments on earlier versions of this paper and L. Powell for the assistance with figures in previous versions. This research was supported by funding from the National Science Foundation Integrative Graduate Education and Research Traineeship (NSF-DGE-0903469) and Nebraska Pheasants and Quail Forever.

Open Access This article is distributed under the terms of the Creative Commons Attribution License which permits any use, distribution, and reproduction in any medium, provided the original author(s) and the source are credited.

\section{Appendix}

Assume the stochastic multivariate Gompertz model as in Eq. 2, but parameterized so that $b_{t}=-\frac{r_{t}}{K_{t}}$.

$$
\begin{aligned}
& \boldsymbol{N}_{t+1}=\boldsymbol{N}_{t} e^{r_{t}+b_{t} \log \left(\boldsymbol{N}_{t}\right)+\varepsilon_{t}} \\
& \boldsymbol{\varepsilon}_{t} \sim \operatorname{MVN}\left(\mathbf{0}, \sum=\sigma_{p}^{2} \boldsymbol{I}_{n}+\rho_{p} \sigma_{p}^{2}\left(\boldsymbol{I}_{n}-\boldsymbol{J}_{n}\right)\right)
\end{aligned}
$$

where $N_{t}$ is the vector of population states for all routes (i.e., separate time series) at time $t$. First, derive $\operatorname{Var}\left(\log \left(\boldsymbol{N}_{t+1}\right)\right)$.

$$
\begin{aligned}
& \operatorname{Var}\left(\log \left(\boldsymbol{N}_{t+1}\right)\right) \\
& =\operatorname{Var}\left(\log \left(\boldsymbol{N}_{t} e^{r_{t}+b_{t} \log \left(\boldsymbol{N}_{t}\right)+\boldsymbol{\varepsilon}_{t}}\right)\right) \\
& =\operatorname{Var}\left(\log \left(\boldsymbol{N}_{t}\right)+r_{t}+b_{t} \log \left(\boldsymbol{N}_{t}\right)+\boldsymbol{\varepsilon}_{t}\right) \\
& =\operatorname{Var}\left(r_{t}+b_{t}+1\right) \log \left(\boldsymbol{N}_{t}\right)+\boldsymbol{\varepsilon}_{t} \\
& =\left(b_{t}+1\right)^{2} \operatorname{Var}\left(\log \left(\boldsymbol{N}_{t}\right)\right)+\operatorname{Var}\left(\boldsymbol{\varepsilon}_{t}\right) \\
& =\left(b_{t}+1\right)^{2} \operatorname{Var}\left(\log \left(\boldsymbol{N}_{t}\right)\right)+\sigma_{p}^{2}\left(1-\rho_{p}\right)
\end{aligned}
$$

Eq. 14 is a first-order linear difference equation with the solution

$$
\begin{aligned}
& \operatorname{Var}\left(\log \left(\boldsymbol{N}_{t}\right)\right) \\
& =\frac{\sigma_{p}^{2}\left(1-\rho_{p}\right)\left(1-\left(1+b_{t}\right)^{2 t}\right)}{1-\left(b_{t}+1\right)^{2}}+\left(b_{t}+1\right)^{2 t} \operatorname{Var}\left(\log \left(\boldsymbol{N}_{0}\right)\right)
\end{aligned}
$$

for $-2<b_{t}<0$. If we assume the initial state $\left(\boldsymbol{N}_{0}\right)$ is known, then $\operatorname{Var}\left(\log \left(\boldsymbol{N}_{0}\right)\right)=0$ and Eq. 16 reduces to:

$=\frac{\sigma_{p}^{2}\left(1-\rho_{p}\right)\left(1-\left(1+b_{t}\right)^{2 t}\right) .}{1-\left(b_{t}+1\right)^{2}}$.

For reasonable $t(t>1)$ and for $b_{t}$ approaching zero:

$$
\approx \frac{\sigma_{p}^{2}\left(1-\rho_{p}\right)}{1-\left(b_{t}+1\right)^{2}}
$$

approximates $\operatorname{Var}\left(\log \left(\boldsymbol{N}_{t}\right)\right)$. After rearranging the denominator and replacing $b_{t}$ with $-\frac{r_{t}}{K_{t}}$, we get Eq. 5:

$$
=\frac{\sigma_{p}^{2}\left(1-\rho_{p}\right)}{2\left(\frac{r_{t}}{K_{t}}\right)-\left(\frac{r_{t}}{K_{t}}\right)^{2}} .
$$

\section{References}

Abrams PA (2002) Will small population sizes warn us of impending extinctions. Am Nat 160:293-305

Baltensperger BH (1987) Hedgerow distribution and removal in nonforested regions of the Midwest. J Soil Water Conserv 42:60-64

Bascompte J (2003) Extinction thresholds: insights from simple models. Ann Zool Fenn 40:99-114

Biggs R, Carpenter SR, Brock WA (2009) Turning back from the brink: detecting an impending regime shift in time to avert it. Proc Natl Acad Sci 106:826-831

Boettiger C, Hastings A (2012a) Quantifying limits to detection of early warning for critical transitions. J R Soc Interface 9:2527-2539

Boettiger C, Hastings A (2012b) Early warning signals and the prosecutor's fallacy. Proc R Soc B 279:4734-4739

Boettiger C, Hastings A (2013) Tipping points: from patters to prediction. Nature 493:157-158

Brooks SP, Gelman A (1998) General methods for monitoring convergence of iterative simulations. J Comput Graph Stat 7:434-455

Burnham KP, Anderson DR (2002) Model selection and multimodel inference, 2nd edn. Springer, Berlin

Carpenter SR, Cole JJ, Pace ML, Batt R, Brock WA, Cline T, Coloso J, Hodgson JR, Kitchell JF, Seekell DA, Smith L, Weidel B (2011) Early warning of regime shifts: a whole-ecosystem experiment. Science 332:1079-1082

Caswell H (2001) Matrix population models: construction analysis and interpretation, 2nd edn. Sinauer, Sunderland

Chen L, Liu R, Liu Z, Li M, Aihara K (2012) Detecting early-warning signals for sudden deterioration of complex diseases by dynamical network biomarkers. Sci Rep 2:342 
Clark JS, Bjørnstad ON (2004) Population time series: process variability, observation errors, missing values, lags and hidden states. Ecology 85:3140-3150

Clark F, Brook BW, Delean S, Reşit Akçakaya H, Bradswa CJA (2010) The theta-logistic is unreliable for modeling most census data. Meth Eco and Evo 1:253-262

Dai L, Vorselen D, Korolev KS, Gore J (2012) Generic indicators for loss of resilience before a tipping point leading to population collapse. Science 336:1175-1177

Dai L, Korolev KS, Gore J (2013) Slower recovery in space before collapse of connected populations. Nature. doi:10.1038/nature12071

Dakos V, van Nes EH, D'Odorico P, Scheffer M (2012) Robustness of variance and autocorrelation as indicators of critical slowing down. Ecology 93(2):264-271

de Valpine P, Hastings A (2002) Fitting population models incorporating process noise and observation error. Ecol Monogr 72:57-76

Dennis B, Ponciano JM, Lele SR, Taper ML, Staples DF (2006) Estimating density dependence, process noise and observation error. Ecol Monogr 76:323-341

Donald PF, Green RE, Heath MF (2001) Agricultural intensification and the collapse of Europe's farmland bird populations. Proc R Soc Lond B Biol Sci 268:25-29

Drake JM, Griffen BD (2010) Early warning signals of extinction in deteriorating environments. Nature 467:456-459

Easterling MR, Ellner SP, Dixon PM (2000) Size-specific sensitivity: applying a new structured population model. Ecology 81:694-708

Efron B, Tibshirani RJ (1993) An introduction to the bootstrap. Chapman and Hall, London

Frank KT, Petrie B, Choi JS, Leggett WC (2005) Trophic cascades in a formerly cod-dominated ecosystem. Science 308:1621-1623

Gelman A, Carlin JB, Stern HS, Rubin DB (2004) Bayesian data analysis, 2nd edn. Chapman and Hall, London

Gompertz B (1825) On the nature of the function expressive of the law of human mortality, and on a new mode of determining life contingencies. Phil Trans R Soc B 115:513-583

Green RE, Cornell SJ, Scharlemann JPW, Balmford A (2005) Farming and the fate of wild nature. Science 307:550-555

Griffen BD, Drake JM (2008) Effects of habitat quality and size on extinction in experimental populations. Proc R Soc B 275:22512256

Hastings A (1996) Population biology: concepts and models. Springer, New York

Hastings A, Wysham DB (2010) Regime shifts in ecological systems can occur with no warning. Ecol Lett 13:464-472

Hefley TJ, Tyre AJ, Blankenship EE (2013) Fitting population growth models in the presence of measurement and detection error. Ecol Model 263:244-250

Hiller T, Powell LA, McCoy TD, Lusk JJ (2009) Long-term agricultural land-use trends in Nebraska, 1866-2007. Great Plains Res 19:225-237

Hoffman JD, Genoways HH, Jones RR (2010) Factors influencing long-term population dynamics of pronghorn (Antilocapra americana): evidence of an Allee effect. J Mammal 91:1124-1134

Hole DG, Whittingham MJ, Bradbury RB, Anderson GQA, Lee PLM, Wilson JD, Krebs JR (2002) Agriculture: widespread local housesparrow extinctions. Nature 418:931-932

Holling CS (1973) Resilience and stability of ecological systems. Annu Rev Ecol Syst 4:1-23

Holmes EE, Sabo JL, Viscido SV, Fagan WF (2007) A statistical approach to quasi-extinction forecasting. Ecol Lett 10:1182-1198

Huang SL, Hao Y, Mei Z, Turvey ST, Want D (2012) Common pattern of population decline for freshwater cetacean species in deteriorating habitats. Freshw Biol 57:1266-1276

International Union for Conservation of Nature (2001) Red list categories and criteria version 3.1. Available at http://www.iucnredlist. org/apps/redlist/static/categories criteria 3 1\#critical. Accessed 28 June 2012
Ives AR, Dakos V (2012) Detecting dynamical changes in nonlinear time series using locally linear state-space models. Ecosphere 3:58

Ives AR, Dennis B, Cottingham KL, Carpenter SR (2003) Estimating community stability and ecological interactions from time-series data. Ecol Monogr 73:301-330

Ives AR, Einarsson A, Jansen VA, Gardarsson A (2008) High-amplitude fluctuations and alternative dynamical states of midges in Lake Myvatn. Nature 452:84-87

Knape J, Jonzen N, Sköld M (2011) On observation distributions for state space models of population survey data. J Anim Ecol 80:1269-1277

Krebs JR, Wilson JD, Bradbury RB, Siriwardena GM (1999) The second silent spring? Nature 400:611-612

Kuehn C (2011) A mathematical framework for critical transitions: bifurcations, fast-slow systems and stochastic dynamics. Physica D 240:1020-1035

Lade SJ, Gross T (2012) Early warning signals for critical transitions: a generalized modeling approach. PLoS Comput Biol 8(2):e1002360

Lande R, Engen S, Sæther B (2003) Stochastic population dynamics. Oxford University Press, Oxford

Lebreton J, Gimenez O (2013) Detecting and estimating density dependence in wildlife populations. J Wildl Manag 77:12-23

Lele SR, Dennis B, Lutscher F (2007) Data cloning: easy maximum likelihood estimation for complex ecological models using Bayesian Markov chain Monte Carlo methods. Ecol Lett 10:551-563

Lele SR, Nadeem K, Schmuland B (2010) Estimability and likelihood inference for generalized linear mixed models using data cloning. J Am Stat Assoc 105:1617-1625

Lenton TM (2011) Early warning of climate tipping points. Nat Clim Chang 1:201-209

Melbourne BA, Hastings A (2008) Extinction risk depends strongly on factors contributing to stochasticity. Nature 454:100-103

Nadeem K, Lele SR (2012) Likelihood based population viability analysis in the presence of observation error. Oikos 121:1656-1664

Pedersen MW, Berg CW, Thygesen UH, Nielsen A, Madsen H (2011) Estimation methods for nonlinear state-space models in ecology. Ecol Model 222:1394-1400

Plummer M (2012) JAGS version 3.3.0 user manual. Available at http:// iweb.dl.sourceforge.net/project/mcmc-jags/Manuals/3.x/jags_user_ manual.pdf

Polansky L, de Valpine P, Loyd-Smith JO, Getz WM (2009) Likelihood ridges and multimodality in population growth rate models. Ecology 90:2313-2320

Ponciano J, Taper ML, Dennis B, Lele SR (2009) Hierarchical models in ecology: confidence intervals, hypothesis testing and model selection using data cloning. Ecology 90:356-362

Ricker WE (1954) Stock and recruitment. J Fish Res Board Can 11:559623

Ringsby TH, Sæther B, Jensen H, Engen S (2006) Demographic characteristics of extinction in a small insular population of house sparrows in northern Norway. Conserv Biol 20:1761-1767

Roseberry JL, Klimstra WC (1984) Population ecology of the bobwhite. Southern Illinois University Press, Carbondale

Roseberry JL, Peterjohn BG, Klimstra WD (1979) Dynamics of an unexploited bobwhite population in deteriorating habitat. J Wildl Manag 43:306-315

Sauer JR, Hines JE, Fallon JE. Pardieck KL, Ziolkowski DJ, Link WA (2011) The North American Breeding Bird Survey, results and analysis 1966-2010, version 12.07.2011. Available at http://www.mbrpwrc.usgs.gov/bbs/bbs.html. Accessed 10 November 2012

Scheffer M, Carpenter SR, Foley JA, Folke C, Walker B (2001) Catastrophic shifts in ecosystems. Nature 413:591-596

Scheffer M, Bascompte J, Brock WA, Brovkin V, Carpenter SR, Dakos V, Held H, van Nes EH, Rietkerk M, Sugihara G (2009) Early warning signals for critical transitions. Nature 461:53-59

Scheffer M, Carpenter SR, Lenton TM, Bascompte J, Brock W, Dakos, van de Koppel J, van de Leemput IA, Levin SA, van Nes EH, 
Pascual M, Vandermeer J (2012) Anticipating critical transitions. Science 338:344-348

Schooler SS, Salau B, Julien MH, Ives AR (2011) Alternative stable states explain unpredictable biological control of Salvinia molesta in Kakadu. Nature 470:86-89

Sólymos P (2010) dclone: data cloning in R. R Journal 2:29-36

Strogatz SH (1994) Nonlinear dynamics and chaos: with applications to physics, biology, chemistry, and engineering. Addison-Wesley, Reading

Stroup WW (2012) Generalized linear mixed models: modern concepts, methods and applications. CRC, Boca Raton

USGS Patuxent Wildlife Research Center (2012) North American Breeding Bird Survey internet data. Available at http://www. pwrc.usgs.gov/bbs/retrieval/. Accessed 16 November 2012

Veech JA (2006) Increasing and declining populations of northern bobwhites inhabit different types of landscapes. J Wildl Manag 70:922-930
Veraart AJ, Faassen EJ, Dakos V, van Nes EH, Lurling M, Scheffer M (2012) Recovery rates reflect distance to a tipping point in a living system. Nature 481:357-359

Wang G (2007) On the latent state estimation of nonlinear population dynamics using Bayesian and non-Bayesian state-space model. Ecol Model 200:521-528

Watkinson AR, Freckleton RP, Robinson RA, Sutherland WJ (2000) Predictions of biodiversity response to genetically modified herbicide-tolerant crops. Science 289:1554-1556

Williams CK (2013) Accounting for wildlife life-history strategies when modeling stochastic density-dependent populations: a review. J Wildl Manag 43:306-315

Williams CK, Ives AR, Applegate RD (2003) Population dynamics across geographical ranges: time-series analyses of three small game species. Ecology 84:2654-2667

Wissel C (1984) A universal law of the characteristic return time near thresholds. Oecologia 65:101-107 